\title{
Effect of Thermal Diffusion and Radiation on Unsteady Mixed Convective Heat and Mass Transfer Flow of a Viscous Fluid in a Vertical Wavy Cylinder
}

\author{
V.Raghavendra Prasad \\ Assistant Professor of Mathematics \\ G.Pulla Reddy Engineering College (Autonomous) \\ Kurnool, A.P., India. \\ vrprasad.knl@gmail.com
}

\author{
M.Siva Sankara Reddy \\ Assistant Professor of Mathematics \\ G.Pulla Reddy Engineering College \\ (Autonomous) Kurnool, A.P., India. \\ msreddy.atp@gmail.com
}

\begin{abstract}
In this paper, we analyze the effect of radiation on the unsteady combined heat and mass transfer of a viscous incompressible fluid in a corrugated pipe in the presence of a constant heat source. The unsteadiness is due to a traveling thermal wave imposed on the boundary. Taking the slope of the boundary wall of the pipe as a perturbation parameter, the equations governing the flow, heat and mass transfer have been solved. The velocity, the temperature and the concentration have been evaluated for different variations of the governing parameters So, N, N1 and $\beta$. The analysis has been carried out in both converging and diverging pipes. The effect of the waviness of the boundary on the flow phenomenon has been exhibited through various profiles of the velocity, the temperature and the concentration. The shear stress, the rate of heat and mass transfer are analyzed computationally for different parameters.
\end{abstract}

Keywords: Radiation effect, heat and mass transfer and heat generating sources

\section{INTRODUCTION}

Transport phenomena involving the combined influence of thermal and concentration buoyancy are often encountered in many Engineering systems and Natural environments. There are many applications of such transport processes in the industry notably in chemical distilleries, heat exchangers, solar energy collectors and thermal protection systems. In all such classes of flows, the driving force is provided by a combination of thermal and chemical diffusion effects. In atmospheric flows thermal convection of the earth by sunlight is affected by differences in water vapor concentration. This buoyancy driven convection due to coupled heat and mass transfer in porous medium has also many important applications in energy related engineering. These include moisture migration, fibrous insulation, spreading of chemical pollution in saturated soils, extraction of geothermal energy and underground disposal of nuclear waste. This problem of combined buoyancy driven thermal and mass diffusion has been studied in parallel plate geometries by a few authors notably Gebhart (8), Lai (15), Chen, Yuh and Moutsoglov (4), Poulikakos (21), Pop et al (20), Angirasa et al (3), Trevisan and Bejan (27). Recently Angirasa et al (3) have presented the analysis of combined heat and mass transfer by natural convection for aiding and opposing buoyancies in fluid saturated porous enclosures.

In most of the studies pertaining to convection flows through the pipes, the axial dependence of the flow variables $(5,7,9,10,16,17$, and 29) is neglected and either the temperature or its gradient is maintained non-uniform on the boundary. Also the heat transfer analysis is investigated in the absence of any internal heat sources in the flow field. The heat transfer in a flow through a pipe in the presence of additional internal heat source has direct application to the modified chemical vapour deposition process. This MCVD process is being used to make high quality optical glass fibres $(25,28$, and 29). In commercialization of this product it is desirable to increase the efficiency of the thermophoretic deposition rate. Since the cost of the fibres depends on the prediction of problems. Keeping these facts in view Krishna et al $(13,14)$ have discussed the combined free and forced convection flow of a viscous incompressible fluid through an axially varying vertical pipe. The problem is analyzed as a regular perturbation problem assuming the slope of the pipe wall to be small. The behaviour of the 
velocity, temperature and heat transfer coefficient is discussed based on numerical computations in detail. Recently Neeraja (18) has investigated the unsteady mixed convection flow in a pipe of varying gap in which the flow is maintained by a prescribed oscillatory flux. And the pipe is maintained at a constant temperature.

In all these studies, the thermal diffusion is not considered. This assumption is true only when the flow takes place at low concentration level. The thermal - diffusion effect (commonly known as Soret Effect) for instances has been utilized for isotope separation and in mixture between gases with very light molecular weight $(\mathrm{H} 2, \mathrm{He})$ and the medium molecular weight $(\mathrm{N} 2$, air) the diffusion-thermo effect was found to be of a magnitude such that it cannot be neglected (6). In view of the importance of this diffusion-thermo effect Jha and Singh (11), Kafoussias (12), Ajay Kumar Singh(2), Rajput et al (22) and Abdul et al (1) have analyzed the convection heat and mass transfer with Soret effect under different conditions. Reddy (25a) has discussed the unsteady double diffusive convective heat transfer flow of a viscous fluid in a vertical wavy pipe.

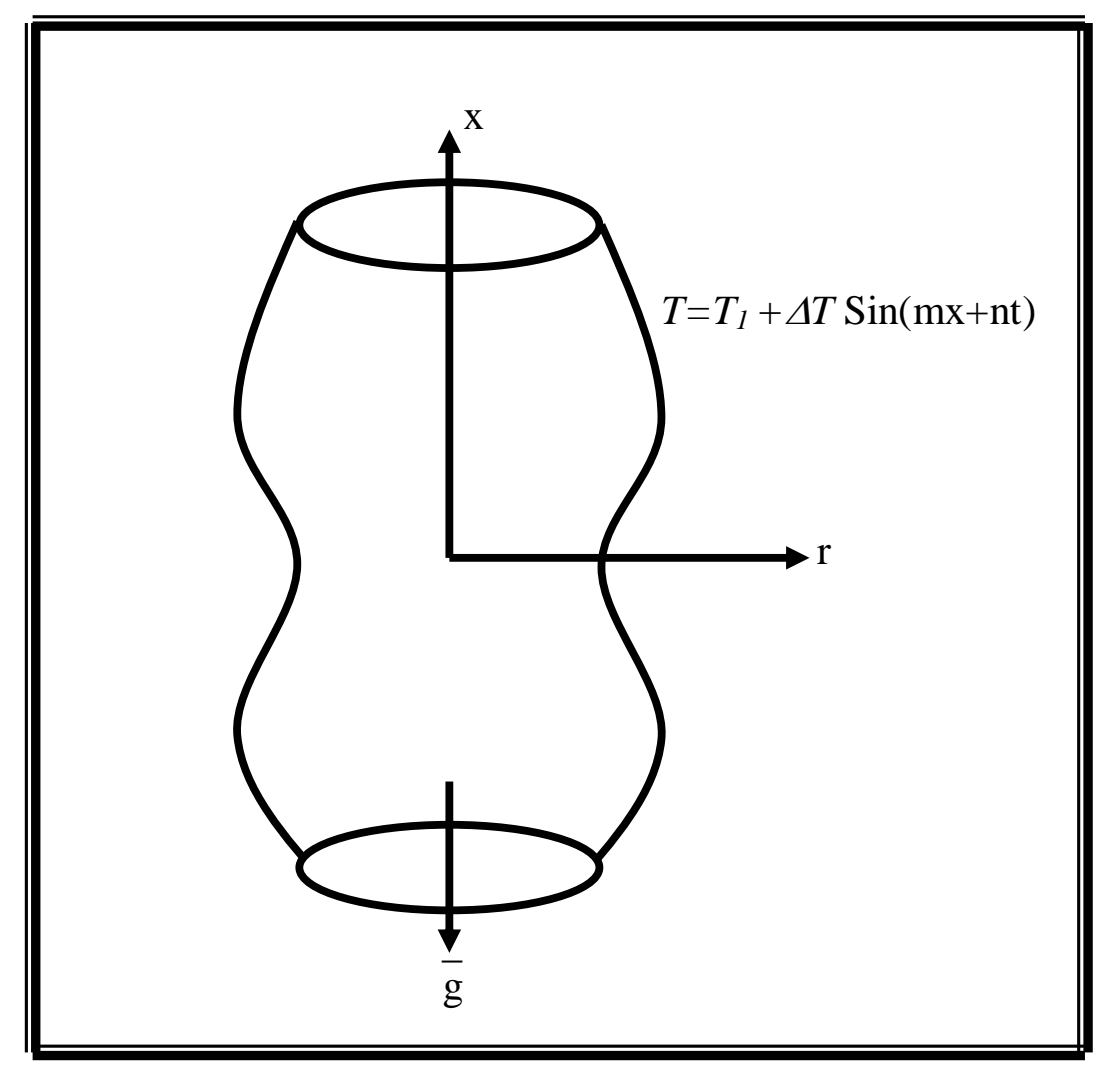

\section{SCHEMATIC DIAGRAM OF THE CONFIGURATION}

\section{FORMUlation}

Consider the unsteady axisymmetric flow of an incompressible, viscous fluid in a vertical pipe of variable cross-section on which a traveling thermal wave is imposed. The Boussinesq approximation is used so that the density variation will be retained only in the buoyancy force. The viscous dissipation is neglected in comparison to the heat flow by convection. The cylindrical polar system $\mathrm{O}(\mathrm{r}, \mathrm{x})$ is choosing with $\mathrm{x}$-axis along the axis of the pipe. The boundary of the pipe is assumed to be

$$
r=a f(\delta x / a)
$$

where ' $\mathrm{a}$ ' is characteristic radial length, $\mathrm{f}$ is a twice differentiable function and ' $\delta$ ' is a small parameter proportional to the boundary slope. The flow is maintained by a constant volume flow rate for which a characteristic velocity $U$ is defined as

$$
U=\frac{2}{a^{2}} \int_{0}^{a f(\delta x / a)} u r d r
$$

The equations governing the flow and heat transfer are 


$$
\begin{gathered}
\rho_{e}\left(\frac{\partial \bar{q}}{\partial t}+\bar{\varsigma} x \bar{q}\right)=-\nabla p+\mu \nabla^{2} \bar{q}-\rho g \\
\nabla \cdot \bar{q}=0 \\
\rho_{e} C_{p}\left(\frac{\partial T}{\partial t}+(\bar{q} \cdot \nabla) T\right)=\lambda \nabla^{2} T+Q-\frac{1}{r} \frac{\partial\left(r q_{R}\right)}{\partial r} \\
\left(\frac{\partial C}{\partial t}+(\bar{q} \cdot \nabla) C\right)=D_{1} \nabla^{2} C+k_{11} \nabla^{2} T \\
\rho=\rho_{e}\left(1-\beta_{1}\left(T-T_{e}\right)-\beta^{*}\left(C-C_{e}\right)\right)
\end{gathered}
$$

Where $\rho_{e}$ is the density of the fluid in the equilibrium state,. $\bar{q}$ is the velocity, $\bar{\zeta}$ is the vorticity, $\mathrm{p}$ is the pressure, T, $\mathrm{C}$ are the temperature and concentration in the flow region, $\rho$ is the density of the fluid, $\mathrm{Cp}$ is the specific heat at constant pressure, $\mathrm{Q}$ is the strength of the heat source, $\lambda$ is the coefficient of thermal conductivity, $\beta_{1}$ is the coefficient of volume expansion, $\beta^{*}$ is the coefficient of expansion with mass fraction, $D_{1}$ is molecular diffusivity, $k_{11}$ is the cross diffusivity and $\mathrm{q}_{\mathrm{r}}$ is the radiative heat flux.

Invoking Rosseland approximation (Brewester (3a)), the radiative heat flux is given by

$$
q_{R}=-\frac{4 \sigma^{\bullet}}{\beta_{R}} \frac{\partial\left(T^{\prime 4}\right)}{\partial r}
$$

and expanding $T^{\prime 4}$ by Taylor's expansion after neglecting higher order terms we get

$$
T^{\prime 4} \cong 4 T_{e}^{3} T^{\prime}-3 T_{e}^{4}
$$

In the equilibrium state,

$$
-\frac{\partial p_{e}}{\partial x}-\rho_{e} g=0
$$

Where $\mathrm{p}=\mathrm{p}_{\mathrm{e}}+\mathrm{p}_{\mathrm{d}}, \mathrm{p}_{\mathrm{d}}$ being the hydrodynamic pressure.

Using equations (2.6) \& (2.9) the equation of momentum (2.2) reduces to

$$
\left(\frac{\partial \bar{q}}{\partial t}+\bar{\varsigma} x \bar{q}\right)=-\nabla p+\mu \nabla^{2} \bar{q}+\beta_{1} g\left(T-T_{e}\right)+\beta^{*} g\left(C-C_{e}\right)
$$

Taking curl on both sides of equation (2.10) and introducing the stream function $\psi$ as

$$
u=-\frac{1}{r} \frac{\partial \psi}{\partial r} \quad, \quad v=\frac{1}{r} \frac{\partial \psi}{\partial x}
$$

where $\mathrm{u}$ is the axial velocity and $\mathrm{v}$ is the radial velocity component the equation in terms of $\psi$ is

$$
\begin{aligned}
\frac{1}{r} \frac{\partial}{\partial t}\left(F^{2} \psi\right)+\frac{1}{r^{2}}\left(\frac{\partial \psi}{\partial r} \frac{\partial\left(F^{2} \psi\right)}{\partial x}\right. & \left.-\frac{\partial \psi}{\partial x} \frac{\partial\left(F^{2} \psi\right)}{\partial r}+\frac{2}{r} \frac{\partial \psi}{\partial x} F^{2} \psi\right) \\
& =\frac{v}{r^{2}} F^{4} \psi-\beta_{1} g\left(T-T_{1}\right)_{r}-\beta^{*} g\left(C-C_{1}\right)_{r}
\end{aligned}
$$

where 


$$
F^{2}=\frac{\partial^{2}}{\partial r^{2}}-\frac{1}{r} \frac{\partial}{\partial r}+\frac{\partial^{2}}{\partial x^{2}}
$$

On introducing the non-dimensional variables

$$
r^{\prime}=\frac{r}{a} \quad, x^{\prime}=m x \quad, t^{\prime}=v m^{2} t, \psi^{\prime}=\frac{\psi}{(U / a)}, \theta=\frac{T-T_{1}}{\Delta T}, C^{\prime}=\frac{C-C_{1}}{\Delta C}
$$

The equations (2.4), (2.5) \& (2.10) (after dropping the dashes) reduce to

$$
\begin{gathered}
\delta^{2} \frac{\partial}{\partial t}\left(E^{2} \psi\right)+\frac{\delta \operatorname{Re}}{r}\left(\frac{\partial \psi}{\partial r} \frac{\partial\left(E^{2} \psi\right)}{\partial x}-\frac{\partial \psi}{\partial x} \frac{\partial\left(E^{2} \psi\right)}{\partial r}+\frac{2}{r} \frac{\partial \psi}{\partial x} E^{2} \psi\right)=E^{4} \psi-\frac{r G}{R_{e}^{r}}\left(\frac{\partial \theta}{\partial r}+N \frac{\partial C}{\partial r}\right) \\
\delta^{2} P \frac{\partial \theta}{\partial t}+\frac{\delta P_{e}}{r}\left(\frac{\partial \theta}{\partial r} \frac{\partial \psi}{\partial x}-\frac{\partial \psi}{\partial r} \frac{\partial \theta}{\partial x}\right)=\left(1+\frac{4}{3 N_{1}}\right)\left(\frac{\partial^{2} \theta}{\partial r^{2}}+\frac{1}{r} \frac{\partial \theta}{\partial r}\right)+\delta^{2} \frac{\partial^{2} \theta}{\partial x^{2}}+\alpha \\
\delta^{2} \frac{\partial \theta}{\partial t}+\frac{\delta R_{e} S c}{r}\left(\frac{\partial C}{\partial r} \frac{\partial \psi}{\partial x}-\frac{\partial \psi}{\partial r} \frac{\partial C}{\partial x}\right)=E^{2} C+\frac{S c S o}{N} E^{2} \theta
\end{gathered}
$$

Where

$$
\begin{aligned}
& E^{2}=\frac{\partial^{2}}{\partial r^{2}}-\frac{1}{r} \frac{\partial}{\partial r}+\delta^{2} \frac{\partial^{2}}{\partial x^{2}} \\
& \delta=m a \quad \text { (Aspect ratio) } \\
& G=\frac{\beta_{1} g \Delta T a^{3}}{v^{2}} \quad \text { (Grashof number) } \\
& R_{e}=\frac{U a}{v} \quad \text { (Reynolds number) } \\
& P=\frac{\mu C_{p}}{\lambda} \quad \text { (Prandtl number) } \\
& P_{e}=R P \quad \text { (Peclect number) } \\
& \alpha=\frac{Q a^{2}}{\lambda} \quad \text { (Heat source parameter) }
\end{aligned}
$$$$
S c=\frac{v}{D_{1}} \quad(\text { Schmidt number })
$$$$
S_{0}=\frac{k_{11} \beta^{\bullet}}{\beta_{1} v} \text { (Soret parameter) }
$$$$
N=\frac{\beta^{\bullet} \Delta C}{\beta \Delta T} \text { (Buoyancy ratio) }
$$$$
N_{1}=\frac{\beta_{R} \lambda}{4 \sigma^{\bullet} T_{e}^{3}} \text { (Radiation parameter) }
$$

Introducing the transformation $\eta=\frac{r}{f(x)}$ the governing equations (2.13)-(2.15) reduce to

$$
\begin{gathered}
\delta f^{2} \frac{\partial}{\partial t}\left(E_{1}^{2} \psi\right)+\frac{\delta f R_{e}}{\eta}\left(\frac{\partial \psi}{\partial \eta} \frac{\partial\left(E_{1}^{2} \psi\right)}{\partial x}-\frac{\partial \psi}{\partial x} \frac{\partial\left(E_{1}^{2} \psi\right)}{\partial \eta}+\frac{2}{\eta} \frac{\partial \psi}{\partial x} E_{1}^{2} \psi\right)=E_{1}^{4} \psi+\frac{G f^{4} \eta}{R_{e}^{2}}\left(\theta_{\eta}+N C_{\eta}\right) \\
\delta^{2} P \frac{\partial \theta}{\partial t}+\delta f P_{e}\left(\frac{\partial \psi}{\partial x} \frac{\partial \theta}{\partial \eta}-\frac{\partial \theta}{\partial x} \frac{\partial \psi}{\partial \eta}\right)=\left(1+\frac{4}{3 N_{1}}\right)\left(\frac{\partial^{2} \theta}{\partial \eta^{2}}+\frac{1}{\eta} \frac{\partial \theta}{\partial \eta}\right)+\delta^{2} f^{2} \frac{\partial^{2} \theta}{\partial x^{2}}+\alpha f^{2}
\end{gathered}
$$


$\delta^{2} S c \frac{\partial C}{\partial t}+\delta f R_{e} S c\left(\frac{\partial \psi}{\partial x} \frac{\partial C}{\partial \eta}-\frac{\partial C}{\partial x} \frac{\partial \psi}{\partial \eta}\right)=\left(\frac{\partial^{2} C}{\partial \eta^{2}}+\frac{1}{\eta} \frac{\partial C}{\partial \eta}\right)+\delta^{2} f^{2} \frac{\partial^{2} C}{\partial x^{2}}+\left(\frac{S c S o}{N}\right) E_{1}^{2} \theta$

where $E_{1}^{2}=\frac{\partial^{2}}{\partial \eta^{2}}-\frac{1}{\eta} \frac{\partial}{\partial \eta}$

We use the asymptotic expansions

$$
\begin{aligned}
& \psi(\eta, x, t)=\psi_{o}(\eta, x, t)+\delta \psi_{1}(\eta, x, t)+\ldots \ldots \ldots . . \\
& \theta(\eta, x, t)=\theta_{o}(\eta, x, t)+\delta \theta_{1}(\eta, x, t)+\ldots \ldots \ldots \\
& C(\eta, x, t)=C_{o}(\eta, x, t)+\delta C_{1}(\eta, x, t)+\ldots \ldots \ldots . .
\end{aligned}
$$

Substituting (2.17) in equations (2.14)-(2.16) and separating the like powers of $\delta$ the equations corresponding to the zeroth order are

$$
\begin{aligned}
& E_{1}^{2} \theta_{0}=-\alpha_{1} f^{2} \\
& E_{1}^{2} C_{0}=-\frac{S c S o}{N} E_{1}^{2} \theta_{0} \\
& E_{1}^{4} \psi_{0}=\frac{G f^{4}}{R_{e}^{2}} \eta\left(\theta_{o, \eta}+N C_{0, \eta}\right)
\end{aligned}
$$

The corresponding conditions on $\psi_{0}, \theta_{0}$ and Co are

$$
\begin{aligned}
& \psi_{0}(\eta, x)=-0.5, \quad \frac{\partial \psi_{0}}{\partial x}=0 \quad, \\
& \theta_{0}=\operatorname{Sin}(x+\gamma t), \quad C_{0}=0 \quad \text { on } \eta=1 \\
& \frac{\partial \theta_{0}}{\partial \eta}=0, \quad \frac{\partial C_{0}}{\partial \eta}=0, \eta \frac{\partial^{2} \psi_{0}}{\partial \eta^{2}}-\frac{\partial \psi_{0}}{\partial \eta}=0, \psi_{0}(\eta, x)=0 \quad \text { on } \eta=0
\end{aligned}
$$

The equations to the first order are

$$
\begin{aligned}
E_{1}^{2} \theta_{1} & =f P_{1}\left(\frac{\partial \psi_{0}}{\partial x} \frac{\partial \theta_{0}}{\partial \eta}-\frac{\partial \theta_{0}}{\partial x} \frac{\partial \psi_{0}}{\partial \eta}\right) \\
E_{1}^{2} C_{1} & =\frac{f R_{e} S c}{\eta}\left(\frac{\partial \psi_{0}}{\partial x} \frac{\partial C_{0}}{\partial \eta}-\frac{\partial C_{0}}{\partial x} \frac{\partial \psi_{0}}{\partial \eta}\right)-\frac{S c S o}{N} E_{1}^{2} \theta_{1}^{2} \\
E_{1}^{2} \psi_{1} & =\frac{G f^{4}}{R_{e}^{2}} \eta\left(\frac{\partial \theta_{1}}{\partial \eta}+N \frac{\partial C_{1}}{\partial \eta}\right)+f R_{e}\left(\frac{\partial \psi_{0}}{\partial \eta} \frac{\partial E_{1}^{2} \psi_{0}}{\partial x}-\frac{\partial \psi_{0}}{\partial x} \frac{\partial E_{1}^{2} \psi_{0}}{\partial \eta}-\frac{2}{\eta} \frac{\partial \psi_{0}}{\partial \eta} E_{1}^{2} \psi_{0}\right)
\end{aligned}
$$

The corresponding boundary conditions are on $\psi_{1}, \theta_{1}$ and $C_{1}$ are

$$
\psi_{1}(\eta, x)=0, \quad \frac{\partial \psi_{1}}{\partial x}=0 \quad, \theta_{1}=0, \quad C_{1}=0 \quad \text { on } \eta=1
$$




$$
\frac{\partial \theta_{1}}{\partial \eta}=0, \quad \frac{\partial C_{1}}{\partial \eta}=0, \eta \frac{\partial^{2} \psi_{1}}{\partial \eta^{2}}-\frac{\partial \psi_{1}}{\partial \eta}=0, \psi_{1}(\eta, x)=0 \quad \text { on } \eta=0
$$

Where

$$
N_{2}=\frac{3 N_{1}}{3 N_{1}+4} \quad P_{1}=P_{e} N_{2} \quad, \alpha_{1}=\alpha N_{2}
$$

\section{SOLUTION}

Solving the coupled equations (2.20)-(2.22) subject to the boundary conditions (2.23), we get the expressions for zeroth order are

$$
\begin{aligned}
& \theta_{o}(\eta, x)=\frac{\alpha f^{2}}{4}\left(1-\eta^{2}\right)+\operatorname{Sin}(x+\gamma t) \\
& C_{0}(\eta, x)=\frac{S c \operatorname{So\alpha } f^{2}}{N}\left(\eta^{2}-1\right) \\
& \psi_{0}=-\frac{A_{1}}{192} \eta^{6}+\frac{A_{2}}{16} \eta^{4}+\frac{A_{4}}{2} \eta^{2}
\end{aligned}
$$

Solving the coupled equations (2.24)-(2.26) subject to the corresponding boundary conditions (2.27) we get the expressions for first order are

$$
\begin{aligned}
\theta_{1}= & \frac{A_{5}}{9}\left(\eta^{3}-1\right)+\frac{A_{6}}{25}\left(\eta^{5}-1\right)+\frac{A_{7}}{49}\left(\eta^{7}-1\right)+\frac{A_{8}}{91}\left(\eta^{9}-1\right) \\
C_{1}= & \frac{A_{9}}{9}\left(\eta^{3}-1\right)+\frac{A_{10}}{25}\left(\eta^{5}-1\right)+\frac{A_{11}}{36}\left(\eta^{6}-1\right)+\frac{A_{12}}{491}\left(\eta^{7}-1\right)+ \\
& +\frac{A_{13}}{64}\left(\eta^{8}-1\right)+\frac{A_{14}}{81}\left(\eta^{9}-1\right)+\frac{A_{15}}{100}\left(\eta^{10}-1\right) . \\
\psi_{1}= & \frac{A_{39}}{192} \eta^{6}+\frac{A_{40}}{630} \eta^{7}+\frac{A_{41}}{1152} \eta^{8}+\frac{A_{42}}{1960} \eta^{9}+\frac{A_{43}}{3840} \eta^{10}+\frac{A_{44}}{6237} \eta^{11}+\frac{A_{45}}{9600} \eta^{12}+ \\
& +\frac{A_{46}}{99 \times 143} \eta^{13}+\frac{A_{47}}{1440 \times 14} \eta^{14}+\frac{A_{48}}{16} \eta^{4}+\frac{A_{49}}{2} \eta^{2}
\end{aligned}
$$

where $\mathrm{A}_{1}, \mathrm{~A}_{2}, \ldots \ldots, \mathrm{A}_{49}$ are the constants.

\section{Shear STress, NuSSElt Number AND SHERWOOd NuMbER}

The shear stress for the motion on the pipe

$$
\sigma_{i j}=-p \delta_{i j}+2 \rho v e_{i j}
$$

where

$$
e_{x x}=\frac{\partial u}{\partial x} \quad, e_{r r}=\frac{\partial v}{\partial r} \quad, e_{r x}=0.5\left(\frac{\partial u}{\partial r}+\frac{\partial v}{\partial x}\right) . .
$$

The shear stress on the pipe $r=f(x)$ in the non-dimensional form is given by

$$
\tau=\left(\sigma_{r x}\left(1-f^{\prime 2}\right)+\left(\sigma_{r r}-\sigma_{x x}\right) f^{\prime}\right) /\left(1+f^{\prime 2}\right)
$$

I terms of the non-dimensional variables, we obtain the non-dimensional shear stress as

$$
\tau=\left(\frac{P}{2 f^{3}}\right)\left(\left(P_{2}\left(\frac{1}{\eta^{2}}\right) \psi_{0, \eta}-\frac{1}{\eta} \psi_{0, \eta \eta}\right)+\varepsilon P_{2}\left(\left(\frac{1}{\eta^{2}}\right) \psi_{1, \eta}-\frac{1}{\eta} \psi_{1, \eta \eta \eta}+2 f f^{\prime}\left(\left(\frac{2}{\eta}\right) \psi_{0, \eta x}-\frac{1}{\eta^{2}} \psi_{0, x}\right)\right)\right.
$$


where $P_{1}=\frac{1}{1+f^{\prime 2}} \quad, P_{2}=1-f^{\prime 2}$

and the corresponding expression is

$$
\tau=-\frac{P_{1} P_{2}}{2 f^{3}}\left(B_{8}+\delta\left(B_{11}-\frac{2 f}{f^{\prime}} B_{10}\right)\right.
$$

The local rate of heat transfer (Nusselt number) on the boundary of the pipe is calculated by using the formula

$$
N u=\frac{1}{f\left(\theta_{m}-\theta_{w}\right)}\left(\frac{\partial \theta}{\partial \eta}\right)_{\eta=1}
$$

where $\quad \theta_{m}=0.5 \int_{0}^{1} \theta d \eta$

and the corresponding expression is, $\quad N u=\frac{\left(B_{3}+\delta B_{4}\right)}{f\left(B_{3}-\operatorname{Sin}\left(D_{1}\right)+\delta B_{2}\right)}$

The local rate of mass transfer (Sherwood number) on the boundary of the pipe is calculated by using the formula

$$
S h=\frac{1}{f\left(C_{m}-C_{w}\right)}\left(\frac{\partial C}{\partial \eta}\right)_{\eta=1}
$$

where $\quad C_{m}=0.5 \int_{0}^{1} \theta d \eta$ and the corresponding expression is

$$
S h=\frac{2\left(B_{5}+\delta B_{6}\right)}{f\left(B_{7}+\delta B_{8}\right)}
$$

where $\mathrm{B}_{1}, \mathrm{~B}_{2}$

$\mathrm{B}_{11}$ are constants.

\section{DiscuSSION OF THE NUMERICAL RESUltS}

In this analysis we discuss the effect of radiation on the unsteady mixed convective heat and mass transfer flow of a viscous fluid in a vertical wavy cylinder on whose wall a traveling thermal wave is imposed. The governing equations are solved by using a regular perturbation technique. The velocity, temperature and concentrations are discussed for different values of $\mathrm{N}$, So, $\mathrm{N}_{1}, \beta$ and $\alpha$.

Fig.1. Variation of $\mathrm{u}$ with $\mathrm{N}$

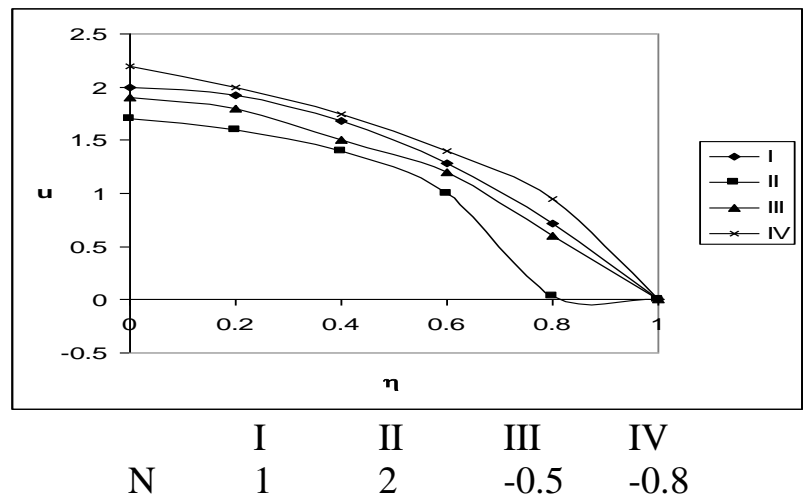

Fig. 2. Variation of $u$ with $S_{0}$

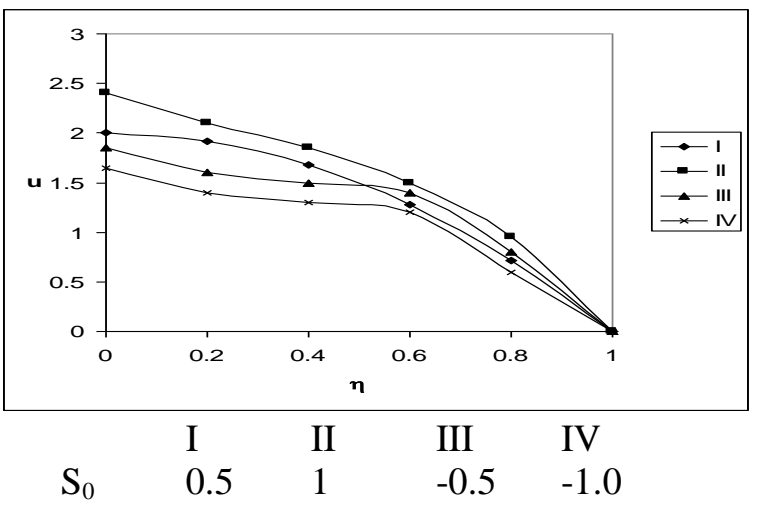


Fig. 1 represents $\mathrm{u}$ with buoyancy ratio $\mathrm{N}$. It is found that when the molecular buoyancy force dominates over the thermal buoyancy force the axial velocity depreciates when the buoyancy forces act in the same directions and for the forces acting in opposite directions $u$ enhances in the flow region. Fig. 2 represents the velocity u enhances with increase in the Soret parameter $\mathrm{S}_{0}>0$ and reduces with $\left|S_{0}\right|$.

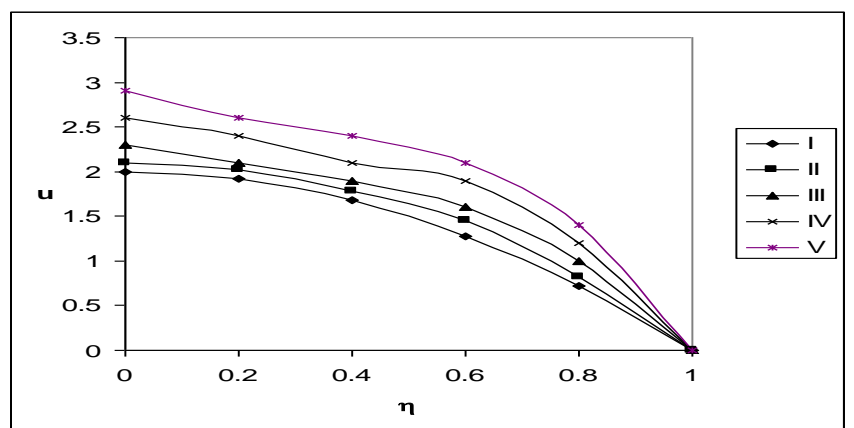

Fig.3. Variation of $\mathrm{u}$ with $\mathrm{N}_{1}$

$\begin{array}{lllll} & \text { I } & \text { II } & \text { III } & \text { IV } \\ \mathrm{N}_{1} & 0.5 & 1.5 & 5 & 10\end{array}$

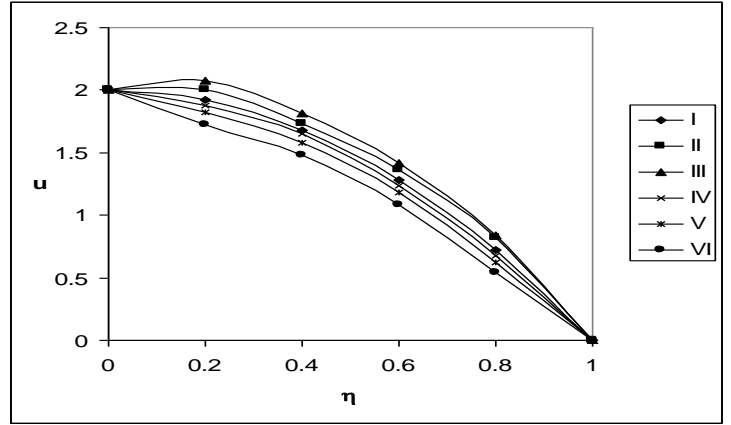

Fig.4. Variation of $\mathrm{u}$ with $\alpha$

$\begin{array}{rrrrrrr}\alpha & \text { I } & \text { II } & \text { III } & \text { IV } & \text { V } & \text { VI } \\ & 2 & 4 & 6 & -2 & -4 & -6\end{array}$

From fig. 3, we find that an increase in the radiation parameter $\mathrm{N}_{1}$ leads to an enhancement in $\mathrm{u}$. The influence of heat sources on $\mathrm{u}$ is shown in fig. 4. It is observed that the axial velocity enhances with increase in $\alpha>0$ and depreciates with $|\alpha|$.

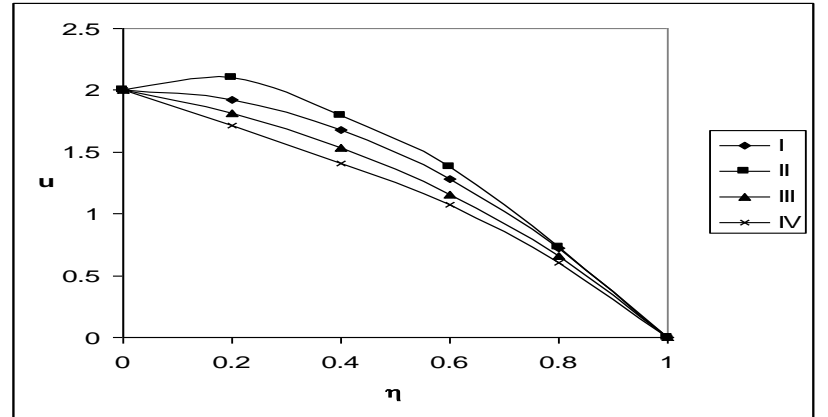

Fig.5. Variation of $\mathrm{u}$ with $\beta$

$\begin{array}{ccccc} & \text { I } & \text { II } & \text { III } & \text { IV } \\ \beta & -0.3 & -0.5 & -0.7 & -0.9\end{array}$

The influence of surface generating on $\mathrm{u}$ is shown in fig. 5. It is found that higher the constriction of the pipe smaller the velocity in the flow region.

From fig. 6, we find that the secondary velocity v reduces with increase in $\mathrm{N}>0$ and enhances with $|\mathrm{N}|$. Fig. 7 represents an increase in $S_{0}>0$ results in an enhancement in $|v|$ and reduces with $\left|S_{0}\right|$.

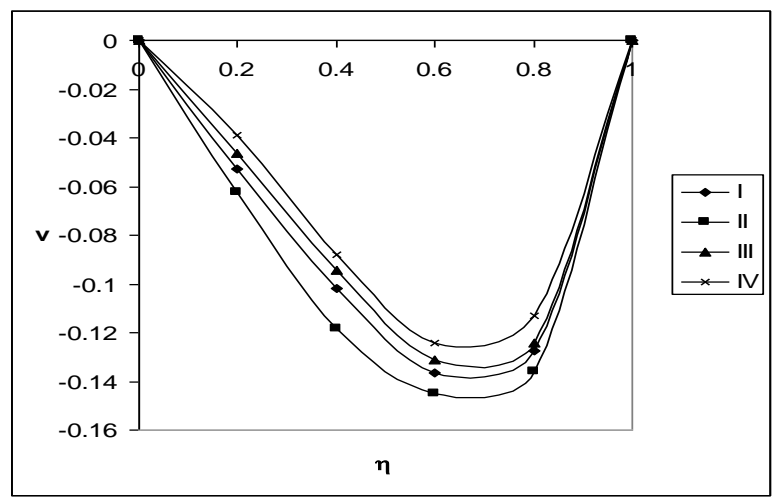

Fig.6. Variation of v with $\mathrm{N}$

$\begin{array}{lllll} & \mathrm{I} & \text { II } & \text { III } & \text { IV } \\ \mathrm{N} & 1 & 2 & -0.5 & -0.8\end{array}$

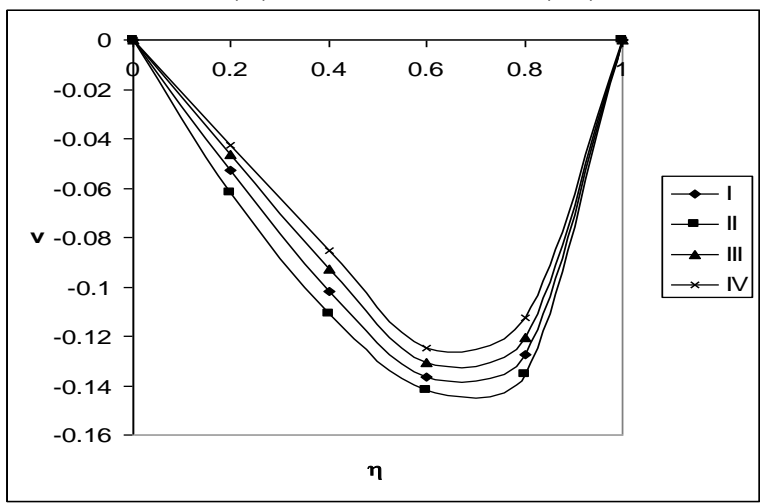

Fig.7. Variation of $\mathrm{v}$ with $\mathrm{S}_{0}$

\begin{tabular}{|c|c|c|}
\hline I & II & III \\
\hline 0.5 & 1 & -0.5 \\
\hline
\end{tabular}

The effect of radiative heat transfer on $\mathrm{v}$ is shown in fig. 8. $|\mathrm{v}|$ experiences depreciation with an increase in the radiation parameter $\mathrm{N}_{1}$. An increase in the strength of heat source/sink results in a marginal depreciation in the secondary velocity is shown in fig.9. 
Effect of Thermal Diffusion and Radiation on Unsteady Mixed Convective Heat and Mass Transfer Flow of a Viscous Fluid in a Vertical Wavy Cylinder

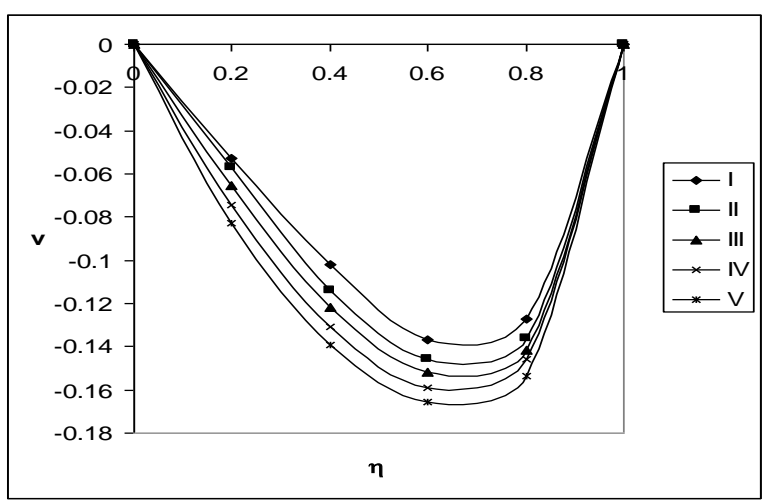

Fig.8. Variation of $\mathrm{v}$ with $\mathrm{N}_{1}$

$\begin{array}{lllll} & \text { I } & \text { II } & \text { III } & \text { IV } \\ \mathrm{N}_{1} & 0.5 & 1.5 & 5 & 10\end{array}$

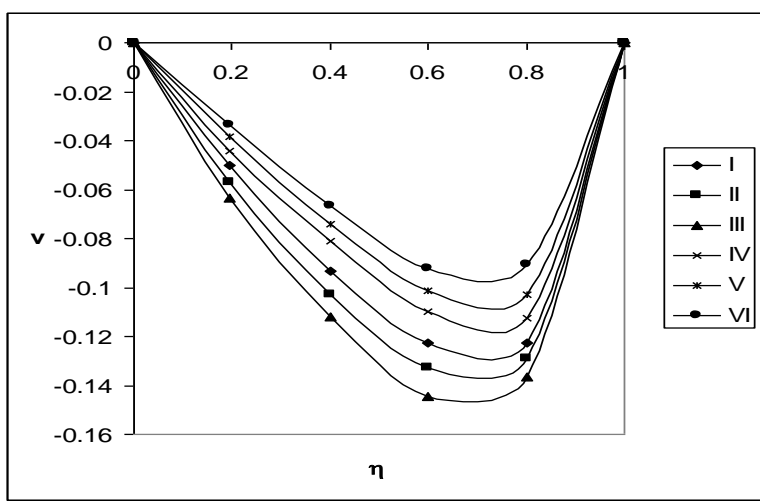

Fig. 9. Variation of $\mathrm{v}$ with $\alpha$

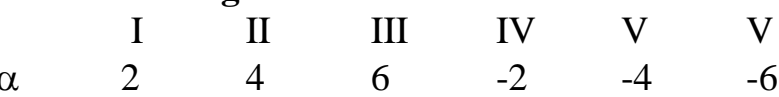

Higher the constriction of the pipe larger $|\mathrm{v}|$ in the flow region is shown in the fig.10.

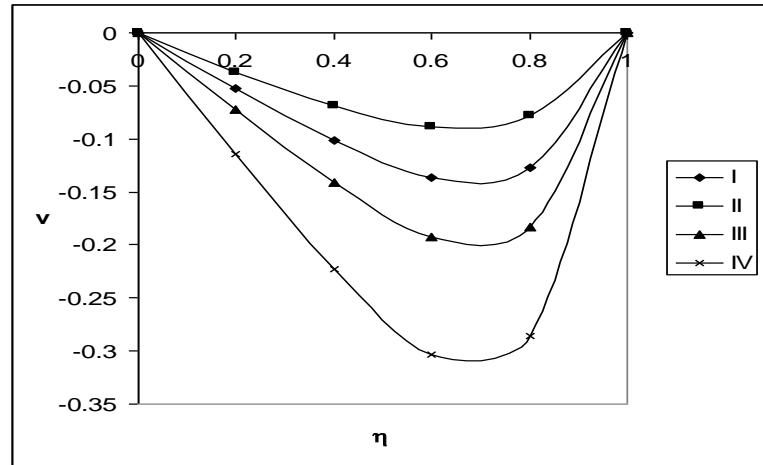

Fig.10. Variation of $v$ with $\beta$

$\begin{array}{ccccc} & \text { I } & \text { II } & \text { III } & \text { IV } \\ \beta & -0.3 & -0.5 & -0.7 & -0.9\end{array}$

Then non-dimensional temperature distribution $(\theta)$ is shown in figs. 11-15 for different values of the governing parameters.

When the molecular buoyancy force dominates over the thermal buoyancy force the actual temperature depreciates when the buoyancy force act in the same direction and for the forces acting in opposite directions if enhances in the flow region is shown in the fig. 11. An increase in the Soret parameter $\left|S_{0}\right|$ results in an enhancement in the actual temperature is shown in the fig. 12.

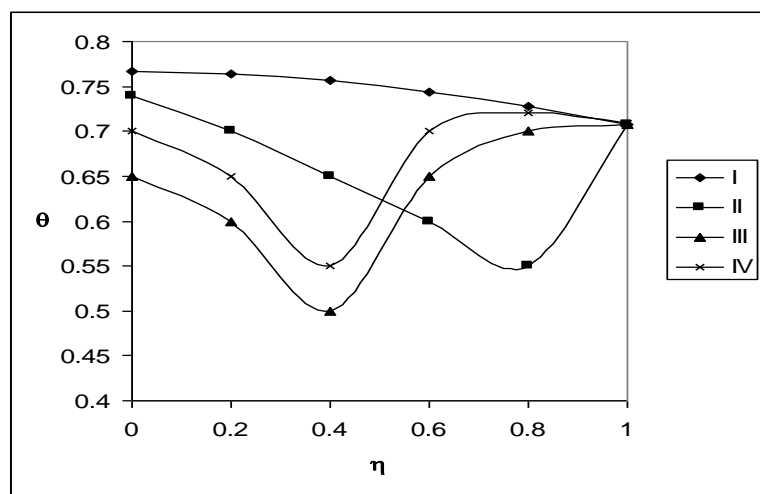

Fig.11. Variation of $\theta$ with $\mathrm{N}$

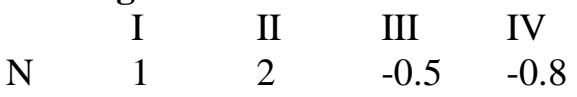

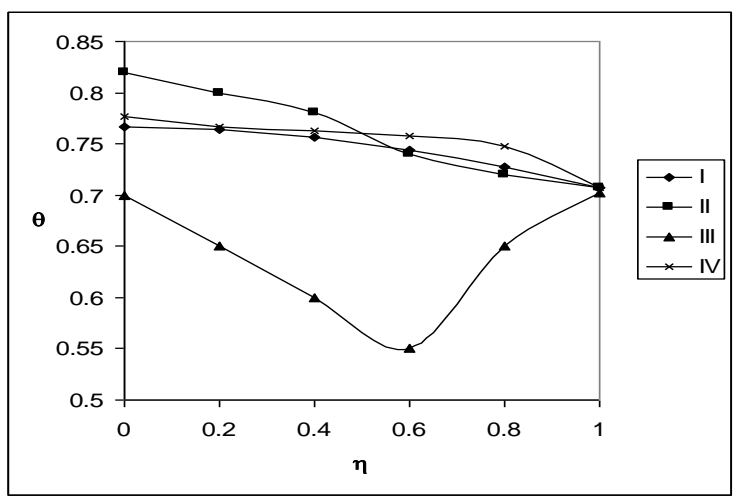

Fig.12. Variation of $\theta$ with $S_{0}$

$\begin{array}{lllll} & \text { I } & \text { II } & \text { III } & \text { IV } \\ \mathrm{S}_{0} & 0.5 & 1 & -0.5 & -1.0\end{array}$


From fig. 13, we find that higher the radiative heat flux larger the actual temperature. From fig. 14, the actual temperature enhances with increase in $\alpha<0$ and reduces with $|\alpha|$.

Fig.13. Variation of $\theta$ with $\mathrm{N}_{1}$

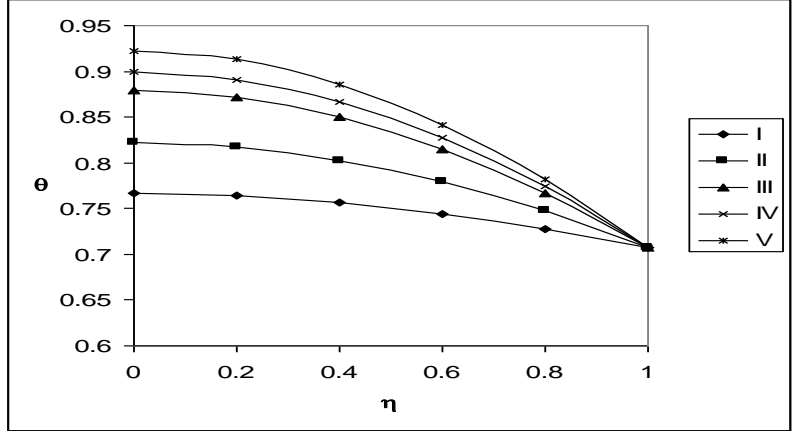

$\begin{array}{llllllllllll} & \text { I } & \text { II } & \text { III } & \text { IV } & & \text { I } & \text { II } & \text { III } & \text { IV } & \text { V } & \text { VI } \\ \mathrm{N}_{1} & 0.5 & 1.5 & 5 & 10 & \alpha & 2 & 4 & 6 & -2 & -4 & -6\end{array}$

Fig.14. Variation of $\theta$ with $\alpha$

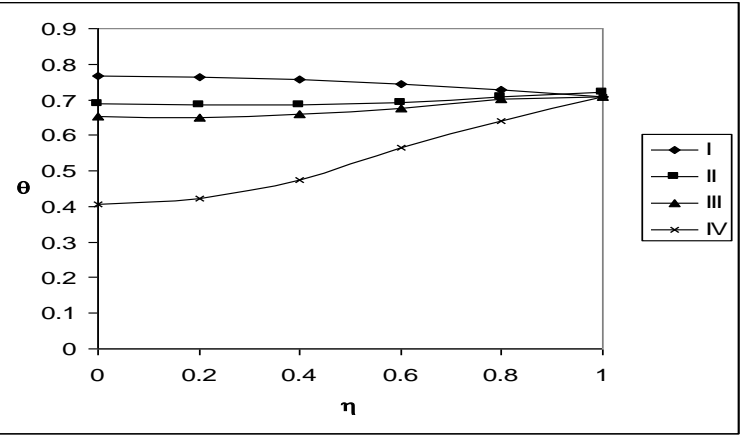

The influence of wall waviness on $\theta$ is shown in fig. 15 . Higher the constriction of the wavy cylinder lesser the actual temperature.

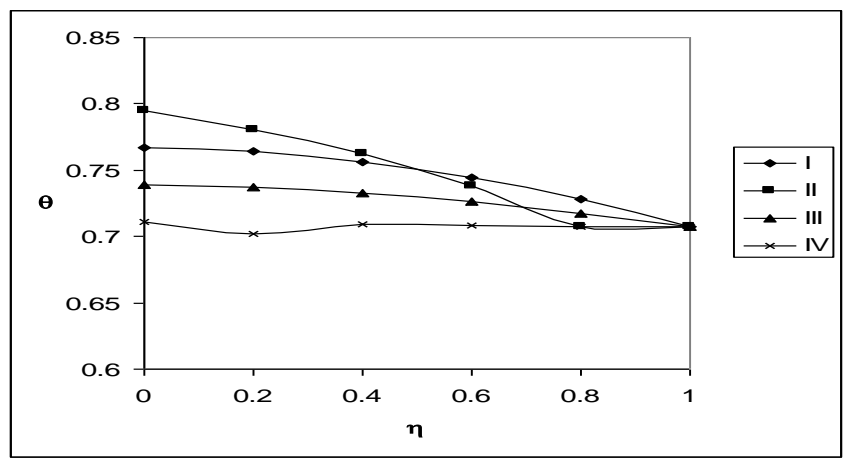

Fig.15. Variation of $\theta$ with $\beta$

$\begin{array}{lllll} & \text { I } & \text { II } & \text { III } & \text { IV } \\ \beta & -0.3 & -0.5 & -0.7 & -0.9\end{array}$
values.

The non-dimensional concentration (C) is shown in figs. 16-19 for different parametric

From fig. 16, the actual concentration enhances with $\mathrm{N}>0$ and reduces with $|\mathrm{N}|$. The actual concentration reduces with increase in $S_{0}<0$ enhances with $|G|$ is shown in fig. 17.

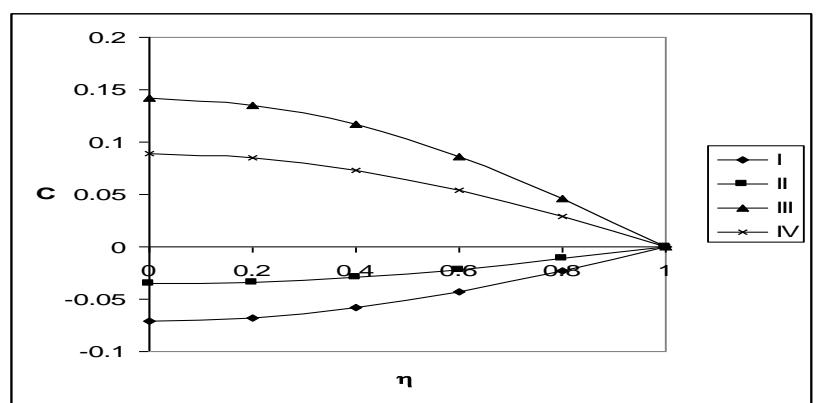

Fig.16. Variation of $\mathrm{C}$ with $\mathrm{N}$

$\begin{array}{lllll} & \mathrm{I} & \text { II } & \text { III } & \text { IV } \\ \mathrm{N} & 1 & 2 & -0.5 & -0.8\end{array}$

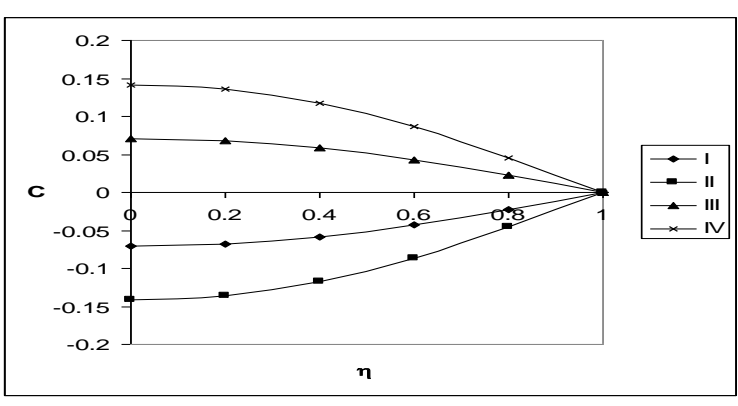

Fig.17. Variation of $\mathrm{C}$ with $\mathrm{S}_{0}$

$$
\text { I II III IV }
$$

1.0From fig. 18, we notice that lesser the radiative heat flux larger the actual concentration. The actual concentration reduces with increase in $\alpha>0$ and enhances with $|\alpha|$ in the entire region is shown in the fig. 19. 
Effect of Thermal Diffusion and Radiation on Unsteady Mixed Convective Heat and Mass Transfer Flow of a Viscous Fluid in a Vertical Wavy Cylinder

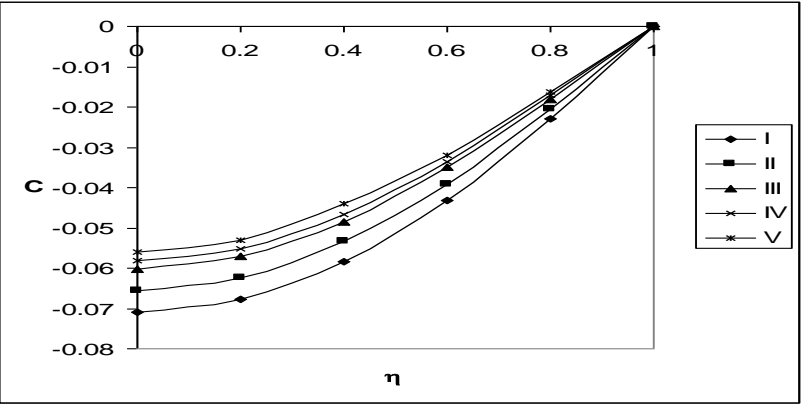

Fig.18.Variation of $\mathrm{C}$ with $\mathrm{N}_{1}$

$\begin{array}{llllllllllll} & \text { I } & \text { II } & \text { III } & \text { IV } & & \text { I } & \text { II } & \text { III } & \text { IV } & \text { V } & \text { VI } \\ \mathrm{N}_{1} & 0.5 & 1.5 & 5 & 10 & \alpha & 2 & 4 & 6 & -2 & -4 & -6\end{array}$

It is found that the stress reduces with increase in $G>0$ and enhances with $G<0$. An increase in $\mathrm{R} \leq 70$ reduces $|\tau|$ and enhances with higher $\mathrm{R} \geq 140$. When the molecular buoyancy force dominates over the thermal buoyancy force the stress experiences an enhancement irrespective of the directions of the buoyancy forces (table. 1). The variation of $\tau$ with heat source parameter $\alpha$ is shown in table. 2 . An increase in $N_{1}$ reduces $|\tau|$ for $G>0$ and enhances it for $G<0$ at $\eta=1$ (table. 3). The influence of surface generating on $\tau$ is shown in table. 4 .

Table-1 : Shear stress $(\tau)$ at $\eta=1$

\begin{tabular}{|c|c|c|c|c|c|c|}
\hline $\mathrm{G}$ & $\mathrm{I}$ & $\mathrm{II}$ & $\mathrm{III}$ & $\mathrm{IV}$ & $\mathrm{V}$ & $\mathrm{VI}$ \\
\hline $10^{3}$ & -15.67979 & -15.36569 & -14.73743 & -15.69979 & -15.65980 & -15.68986 \\
\hline $3 \times 10^{3}$ & -15.67952 & -15.551 & -14.73729 & -15.66953 & -15.64953 & -15.65253 \\
\hline$-10^{3}$ & -15.68007 & -15.36589 & -14.73758 & -15.4007 & -15.66007 & -15.67027 \\
\hline$-3 \times 10^{3}$ & -15.68037 & -15.36608 & -14.73772 & -15.74037 & -15.68980 & -15.64360 \\
\hline $\mathrm{R}$ & 35 & 70 & 140 & 35 & 35 & 35 \\
\hline $\mathrm{N}$ & 1 & 1 & 1 & 2 & -0.5 & -0.8 \\
\hline
\end{tabular}

Table-2 : Shear stress $(\tau)$ at $\eta=1$

\begin{tabular}{|c|c|c|c|c|c|c|}
\hline $\mathrm{G}$ & $\mathrm{I}$ & $\mathrm{II}$ & $\mathrm{III}$ & $\mathrm{IV}$ & $\mathrm{V}$ & $\mathrm{VI}$ \\
\hline $10^{3}$ & -15.67979 & -15.67961 & -15.67935 & -15.67998 & -15.6799 & -15.67990 \\
\hline $3 \times 10^{3}$ & -15.67952 & -15.67903 & -15.67845 & -15.67007 & -15.68005 & -15.67983 \\
\hline$-10^{3}$ & -15.68007 & -15.68027 & -15.68055 & -15.67989 & -15.67996 & -15.69997 \\
\hline$-3 \times 10^{3}$ & -15.68037 & -5.68102 & -15.68198 & -15.67980 & -15.67972 & -15.68003 \\
\hline$\alpha$ & 2 & 4 & 6 & -2 & -4 & -6 \\
\hline
\end{tabular}

Table-3 : Shear stress $(\tau)$ at $\eta=1$

\begin{tabular}{|c|c|c|c|c|}
\hline $\mathrm{G}$ & $\mathrm{I}$ & II & III & IV \\
\hline $10^{3}$ & -15.67996 & -15.67973 & -15.67972 & -15.67970 \\
\hline $3 \times 10^{3}$ & -15.67943 & -15.67934 & -15.67930 & -15.67927 \\
\hline$-10^{3}$ & -15.68011 & -15.68015 & -15.68016 & -15.68017 \\
\hline$-3 \times 10^{3}$ & -15.68048 & -15.68059 & -15.68063 & -15.68068 \\
\hline $\mathrm{N}_{1}$ & 1.5 & 5 & 10 & 100 \\
\hline
\end{tabular}

Table-4 : Shear stress $(\tau)$ at $\eta=1$

\begin{tabular}{|c|c|c|c|}
\hline G & I & II & III \\
\hline $10^{3}$ & -5.67008 & -15.67983 & -73.14547 \\
\hline $3 \times 10^{3}$ & -5.66960 & -15.67962 & -73.14543 \\
\hline$-10^{3}$ & -5.67091 & -15.68004 & -73.14551 \\
\hline$-3 \times 10^{3}$ & -5.67210 & -15.68026 & -73.14555 \\
\hline$\beta$ & -0.3 & -0.5 & -0.7 \\
\hline
\end{tabular}


It is found that the rate of heat transfer at $\eta=1$ reduces with increase in $G>0$ and enhances with $G<0$. An increase in $R$ leads to a depreciation in $\tau$. When the molecular buoyancy force dominates over the thermal buoyancy force the rate of heat transfer enhances irrespective of the buoyancy forces directions of (table. 5). The variation of $\mathrm{Nu}$ with heat source parameter $\alpha$ is shown in table 6. Also $|\mathrm{Nu}|$ experiences an enhancement with increase in the radiation parameter $\mathrm{N}_{1}$ (table 7). From table.8, we find that higher the constriction of the wavy pipe larger $|\mathrm{Nu}|$ at $\eta=1$.

Table-5 : Nusselt Number $(\mathrm{Nu})$ at $\eta=1$

\begin{tabular}{|c|c|c|c|c|c|c|}
\hline $\mathrm{G}$ & $\mathrm{I}$ & $\mathrm{II}$ & $\mathrm{III}$ & $\mathrm{IV}$ & $\mathrm{V}$ & $\mathrm{VI}$ \\
\hline $10^{3}$ & -9.7994 & -8.01153 & -5.28079 & -9.81946 & -9.7849 & -9.7742 \\
\hline $3 \times 10^{3}$ & -9.79825 & -8.01062 & -528018 & -9.89012 & -9.7742 & -9.7659 \\
\hline$-10^{3}$ & -9.80056 & -8.01245 & -5.28139 & -9.8102 & -9.7956 & -7.7852 \\
\hline$-3 \times 10^{3}$ & -9.80171 & -8.01336 & -5.28199 & -9.8112 & -9.8007 & -9.7986 \\
\hline $\mathrm{R}$ & 35 & 70 & 140 & 35 & 35 & 35 \\
\hline $\mathrm{N}$ & 1 & 1 & 1 & 2 & -0.5 & -0.8 \\
\hline
\end{tabular}

Table-6 : Nusselt Number $(\mathrm{Nu})$ at $\eta=1$

\begin{tabular}{|c|c|c|c|c|c|c|}
\hline $\mathrm{G}$ & $\mathrm{I}$ & $\mathrm{II}$ & $\mathrm{III}$ & $\mathrm{IV}$ & $\mathrm{V}$ & VI \\
\hline $10^{3}$ & -9.7994 & -10.83924 & -11.21132 & -14.77049 & -13.30063 & -12.84987 \\
\hline $3 \times 10^{3}$ & -9.79825 & -10.83761 & -11.20929 & -14.76954 & -13.30026 & -12.84995 \\
\hline$-10^{3}$ & -9.80056 & -10.84087 & -11.21335 & -14.77143 & -13.30101 & -12.84979 \\
\hline$-3 \times 10^{3}$ & -9.80171 & -10.84256 & -11.21538 & -14.77238 & -13.30138 & -12.84971 \\
\hline$\alpha$ & 2 & 4 & 6 & -2 & -4 & -6 \\
\hline
\end{tabular}

Table-7 : Nusselt Number (Nu) at $\eta=1$

\begin{tabular}{|c|c|c|c|c|c|}
\hline $\mathrm{G}$ & $\mathrm{I}$ & II & III & IV & V \\
\hline $10^{3}$ & -9.7994 & -8.10763 & -6.67480 & -6.21885 & -5.73624 \\
\hline $3 \times 10^{3}$ & -9.79825 & -8.10542 & -6.67164 & -6.21540 & -5.73248 \\
\hline$-10^{3}$ & -9.80056 & -8.10984 & -6.67796 & -6.22230 & -5.73999 \\
\hline$-3 \times 10^{3}$ & -9.80171 & -8.11205 & -6.68111 & -6.22231 & -5.74376 \\
\hline $\mathrm{N}_{1}$ & 1.5 & 2.5 & 5 & 10 & 100 \\
\hline
\end{tabular}

Table-8 :Nusselt Number (Nu) at $\eta=1$

\begin{tabular}{|c|c|c|c|}
\hline $\mathrm{G}$ & $\mathrm{I}$ & $\mathrm{II}$ & $\mathrm{III}$ \\
\hline $10^{3}$ & -7.7600 & -9.7994 & -10.92405 \\
\hline $3 \times 10^{3}$ & -7.75667 & -9.79825 & -10.92446 \\
\hline$-10^{3}$ & -7.76333 & -9.80056 & -10.92484 \\
\hline$-3 \times 10^{3}$ & -7.7666 & -9.80171 & -10.92503 \\
\hline$\beta$ & -0.3 & -0.5 & -0.7 \\
\hline
\end{tabular}

We find that the rate of mass transfer at $\eta=1$ reduces with $G>0$ and enhances with $G<0$. An increase in $\mathrm{R}$ results in depreciation in $|\mathrm{Sh}|$. From table 9, the rate of heat transfer reduces with $\mathrm{N}$ when the buoyancy forces act in the same direction and for the forces acting in opposite direction $|\mathrm{Sh}|$ enhances at $\eta=1$. $|S h|$ enhances with $S_{0}>0$ and reduces with $\left|S_{0}\right|(<0)$ (table. 10). Higher the constriction the wavy pipe larger $|\mathrm{Sh}|$ and for further higher $|\beta| \geq 0.7$, smaller $|\mathrm{Sh}|$ at $\eta=1$ (table. 11). Higher the constriction the wavy pipe larger $|\mathrm{Sh}|$ and for further higher $|\beta| \geq 0.7$, smaller $|\mathrm{Sh}|$ at $\eta=1$ (table. 12).

Table-9 : Sherwood Number (Sh) at $\eta=1$

\begin{tabular}{|c|c|c|c|c|c|c|}
\hline $\mathrm{G}$ & $\mathrm{I}$ & II & III & IV & V & VI \\
\hline $10^{3}$ & 14.84282 & 7.56577 & -3.6435 & 14.79147 & 14.92052 & 14.93616 \\
\hline $3 \times 10^{3}$ & 14.8405 & 7.56389 & -3.64507 & 14.78876 & 14.91879 & 14.93454 \\
\hline$-10^{3}$ & 14.84515 & 7.56766 & -3.64197 & 14.79418 & 14.92225 & 14.93777 \\
\hline$-3 \times 10^{3}$ & 14.84747 & 7.56954 & -3.64042 & 14.7969 & 14.92399 & 14.93938 \\
\hline $\mathrm{R}$ & 35 & 70 & 140 & 35 & 35 & 35 \\
\hline $\mathrm{N}$ & 1 & 1 & 1 & 2 & -0.5 & -0.8 \\
\hline
\end{tabular}


Effect of Thermal Diffusion and Radiation on Unsteady Mixed Convective Heat and Mass Transfer Flow of a Viscous Fluid in a Vertical Wavy Cylinder

Table-10 : Sherwood Number (Sh) at $\eta=1$

\begin{tabular}{|c|c|c|c|c|c|c|}
\hline $\mathrm{G}$ & $\mathrm{I}$ & $\mathrm{II}$ & $\mathrm{III}$ & $\mathrm{IV}$ & $\mathrm{V}$ & $\mathrm{VI}$ \\
\hline $10^{3}$ & 14.84282 & 19.16302 & 20.7118 & 35.59484 & 29.43143 & 27.54543 \\
\hline $3 \times 10^{3}$ & 14.8405 & 19.1597 & 20.70753 & 35.5933 & 29.43083 & 27.54556 \\
\hline$-10^{3}$ & 14.84515 & 19.16634 & 20.71607 & 35.59639 & 29.43203 & 27.54556 \\
\hline$-3 \times 10^{3}$ & 14.84747 & 19.16965 & 20.72033 & 35.59793 & 29.43263 & 27.54519 \\
\hline$\alpha$ & 2 & 4 & 6 & -2 & -4 & -6 \\
\hline
\end{tabular}

Table-11 : Sherwood Number (Sh) at $\eta=1$

\begin{tabular}{|c|c|c|c|c|c|}
\hline $\mathrm{G}$ & $\mathrm{I}$ & $\mathrm{II}$ & $\mathrm{III}$ & $\mathrm{IV}$ & $\mathrm{V}$ \\
\hline $10^{3}$ & 13.47297 & 14.84282 & 11.99236 & 11.43895 & 10.79985 \\
\hline $3 \times 10^{3}$ & 13.47177 & 14.8405 & 11.99276 & 11.44001 & 10.80167 \\
\hline$-10^{3}$ & 13.47417 & 14.84515 & 11.99195 & 11.44001 & 10.80167 \\
\hline$-3 \times 10^{3}$ & 13.47537 & 14.84747 & 11.99155 & 11.43790 & 10.79620 \\
\hline $\mathrm{N}_{1}$ & 1.5 & 2.5 & 5 & 10 & 100 \\
\hline
\end{tabular}

Table-12 : Sherwood Number (Sh) at $\eta=1$

\section{Conclusion}

\begin{tabular}{|c|c|c|c|}
\hline $\mathrm{G}$ & $\mathrm{I}$ & $\mathrm{II}$ & $\mathrm{III}$ \\
\hline $10^{3}$ & 13.67022 & 14.84282 & 9.17279 \\
\hline $3 \times 10^{3}$ & 13.66823 & 14.8405 & 9.17231 \\
\hline$-10^{3}$ & 13.67221 & 14.84515 & 9.17327 \\
\hline$-3 \times 10^{3}$ & 13.67419 & 14.84747 & 9.17375 \\
\hline$\beta$ & -0.3 & -0.5 & -0.7 \\
\hline
\end{tabular}

From the studies made on this paper, we conclude that,

When the molecular buoyancy force dominates over the thermal buoyancy force, the axial velocity depreciates. When the buoyancy forces act in the same directions and for the forces acting in opposite directions, velocity enhances in the flow region. And the velocity enhances with increase in the Soret parameter.

\section{ACKNOWLEDGEMENTS}

I thank to Dr. D.R.V.Prasada Rao, Professor, Dept. of Mathematics, S.K.University, Anantapur for his guidance and valuable suggestions.

\section{REFERENCES}

[1] Abdul Sattar.Md and Mahmud Alam.Md :Thermal diffusion as well as transpiration effects on MHD free convection and Mass transfer flow past an accelerated vertical porous plate : Ind.J.Pure and Appl. Maths.,V.25,No.6,pp.679-688 (1994)

[2] Ajay Kumar singh : MHD free convection and Mass transfer flow with Hall effect,viscous dissipation, Joule heating and thermal diffusion. Ind.J.Pure and appl. Maths.,V.41,pp.24-35 (2003)

[3] Angirasa.D and Peterson.G.P and Pop.I : Combined heat and mass transfer by natural convection with opposing buoyancy effects in a fluid saturated porous medium. Int.J.Heat Mass Transfer, V.40, No. 12 , pp.2755-2773 (2004)

[3a] Brewester. M.Q. : Thermal radiative transfer and properties, John Wiley \& Sons Inc., New York, 1992

[4] Chen,T.S .,Yuh,C.F and Moutsoglov,H: Combined heat and mass transfer in mixed convection along vertical and inclined plantes. Int.J.Heat Mass Transfer,V.23, pp.527- 537 (1980)

[5] Dephew.C.A and August.S.E: Trans.Amer,Soc.Mech.Engg, Series C,J, Heat Transfer, V.93,p.350 (1971)

[6] Eckert.L.R.G and Drake.R.M : Analysis of heat and mass transfer, McGraw-Hill Book Co, New York (1972) 
[7] Fagri.M and Welty.J.R :Int.J.Heat Mass Transfer,V.21,p.317 (1978)

[8] Gebhart.B, Jaluria.Y, Mahajan .R.L and Sammakia.B : Buoyancy induced flows and transport. Ch.15, Hemi Sphere, Washington, Oc.(1988)

[9] Gill.W.M and Casal.A.D : A theoretical Investigation of natural convection effects in forced horizontal flows. Ame.Chem.Eng.Jour.,(1962)

[10] Heinbochal.J.H and Ash.R.L : ZAMP,V.21,p.266 (1970)

[11] Jha.B.K and Sngh.A.K : Astro.Phys.Space Science,V.173.p.251 (1990)

[12] Kafoussias.N.G : Astro.Phys.Space Sci.,V.192, p.11 (1992)

[13] Krishna.D.V and Pasad Rao.D.R.V :Proceedings of the American Society of Mechanical Engineers,U.S.A.,pp.1-9 (1987)

[14] Krishna.D.V .,Rao.D.R.V and Sarojamma.G: Mixed thermal convection in a cylinder of nonuniform gap .,Int.comm..Heat Mass Transfer,V.16, pp.237-246 (1989)

[15] Lai,F.J: Coupled heat and mass transfer by natural convection from a horizontal line source in a saturated porous medium,Int.comm..Heat Mass Transfer,V.17,pp.489-499 (1990)

[16] Morton.B.R : Q.J.Mech.Appl.Maths.,V.12, p.410 (1959)

[17] Morton.B.R : J.Fluid mech.,V.B., P.227(1960)

[18] Neeraja.G : Ph.D thesis,S.P.Mahila University, Tirupathi, India (1993)

[19] Neild.D.A and Bejan.B : Convection in porous media,Ch.9,Springer-verlag, New-York (1992)

[20] Pop.I and Herwing.H : Transient mass transfer from an isothermal vertical flat plateembedded in a porous medium, Int. .Comm. Heat Mass transfer, V.17, pp.813-821(1990)

[21] Poulikakos.D : On buoyancy induced heat and mass transfer from a concentrated source in an infinite porous medium. Int.J.Heat Mass Transfer, V.28,No.3,pp.621-629 (1985)

[22] Rajput.U.S ,Varshney.N.K and Debangana Rajput : Thermo diffusion on MHD free convection flow of a viscous fluid with dissipation and mass transfer. Acta Ciencia Indica,V.xxix, No.2, p.251(2003)

[23] Raptis.A.A : Effect of Mass transfer on the hydromagnetic flow past a vertical limiting surface. Astro Physics and Space Science, V.92, pp.135-142 (1983)

[24] Reynods.W.C : Trans.Ame.Soc.Mech.Engs.Ser,C-J.Heat Transfer,v.82, p.108 (1960)

[25] Simpkins,P.G,Greenberg-Kosinski's and Maechsney,J.B: Thermophoresis:The mass transfer mechanism in modified chemical vapour deposition. J.Appl.Phys., V.50(9).P.5676 (1979)[25a] Sreenivasa reddy.B : Thermo-diffusion effect on convective heat and mass transfer through a porous medium, Ph.D. Thesis (2006), S.K.University, Anantapur.

[26] Trevisan.O.V and Bejan.A: Mass and Heat transfer by high Rayleigh number convection in a porous medium heated from below. Int.J.Heat Mass Transfer,V.39, pp.2241-2356(1987)

[27] Trevisan.O.V and Bejan.A : Mass and Heat transfer by natural convection in a vertical slot filled with porous medium. Int.J.Heat Mass Transfer,V.29, pp.403-415(1986)

[28] Waller. K.L, George.M, Mogsy and Franz.T, Geyling : J.Collod interface Sci. V.69(1), p.138 (1979)

[29] Walker.K, Lgeyling.F.T and Nagel.S.R : J.Ame.ceram.Sci.,V.63(9), pp.552 (1980) 


\section{AUTHOR'S BIOGRAPHY}

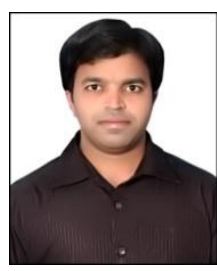

Dr. V. Raghavendra Prasad, presently working as an Assistant Professor of Mathematics in Basic Engineering Department in G.Pulla Reddy Engineering College (Autonomous), Kurnool, Andhra Pradesh, India. He obtained his Ph.D. degree in 2012 from S.K. University, Anantapur, A.P., India. He has presented papers in International and National Conferences and seminars and published papers in popular International journals for his credit. He has 8 years of teaching experience.

Dr. M. Siva Sankara Reddy, presently working as an Assistant Professor of Mathematics in Basic Engineering Department in G.Pulla Reddy Engineering College (Autonomous), Kurnool, Andhra Pradesh, India. He obtained his Ph.D. degree in 2008 from S.K. University, Anantapur, A.P., India. He has presented papers in National and International conferences and seminars and published papers in International journals. He has 10 years of teaching experience. His area of interest is Fluid Dynamics. 